\title{
$\nabla$
}

\section{Dermoscopic and clinical features of head and neck melanoma*}

\author{
Fatma Pelin Cengiz ${ }^{1}$ \\ Nazan Emiroglu ${ }^{1}$ \\ Rainer Hofmann Wellenhof ${ }^{4}$
}

\author{
Abdurrahman Bugra Cengiz ${ }^{2}$ \\ Ela Comert ${ }^{3}$
}

DOI: http://dx.doi.org/10.1590/abd1806-4841.20153341

\begin{abstract}
BACKGROUND: The dermoscopic criteria of extrafacial melanomas are well-known.
Овлестіvе: To determine the frequency of dermatoscopic findings in head and neck melanomas (HNM) and to assess the distinguishing dermoscopic criteria of facial and extrafacial melanoma.

MetHODS: This observational study included 108 patients with HNM (63\% male, mean age 64 years). Participants underwent individual dermoscopic imaging of clinically melanoma. All lesions were excised, and histopathological examination was performed on all specimens.

RESULTS: Drawing on histopathological analysis, lentigo maligna melanoma or lentigo maligna was diagnosed in 60 lesions, superficial spreading melanoma in 18, nodular in 10, desmoplastic in 8, superficial spreading melanoma in situ in 12. The most frequent location for head and neck melanoma was the cheek (60 patients, 55.6\%). Eight prominent dermatoscopic features were observed in facial melanoma: annular-granular pattern (18\%); rhomboidal structures (29\%); pseudonetwork (29\%); asymmetrical, pigmented, follicular openings (51\%); obliterated hair follicles (8\%); red rhomboidal structures (18\%); increased density of the vascular network (32\%); scar-like depigmentation (59\%).

Conclusions: HNM has specific dermoscopic features, and classical extrafacial dermoscopic rules are less useful for diagnosis of facial melanoma. In our study, further characteristic dermatoscopic findings were detected in facial melanoma such as low frequencies of irregular dots, 2 or fewer colors in lesions, the presence of pseudonetwork, increased density of the vascular network, red rhomboidal structures, in addition to dermatoscopic findings of extrafacial melanoma. Thus, it is concluded that the prediction and identification of HNM may be evident with the help of these signs.
\end{abstract}

Keywords: Dermoscopy; Head and neck neoplasms; Melanoma

\section{INTRODUCTION}

Cutaneous melanoma is an aggressive malignant tumor of melanocytes, accounting for $5 \%$ of all skin cancer and $65 \%$ of skin cancer deaths. ${ }^{1}$ Incidence rates are increasing by $5 \%$ each year. It has been estimated that $20 \%$ of melanomas arise from the head and neck region, of which $70-90 \%$ occur in the facial region, $7 \%$ in the neck, $7 \%$ in the external ear, and 3\% in the scalp. 2,3 Melanomas of the head and neck affect more men than women, unlike melanomas on other sites. ${ }^{4}$ Head and neck melanomas (HNM) have a poorer prognosis than melanomas of the trunk and extremities. The estimated five-year overall survival rate is reportedly $74 \%$ for head and neck melanomas, $84 \%$ for extremity melanoma and 82\% for trunk melanoma. ${ }^{5}$ Prognosis for melanoma is dependent on the stage of disease. Since current therapies are not very effective at advanced stages, removal of the tumor at early stages with adequate surgical margins is valuable to patients with malignant melanoma. Hence, early detection of melanoma is critical in decreasing mortality rates and

Received on 24.12.2013.

Approved by the Advisory Board and accepted for publication on 05.08.2014.

Work performed at the Kars State Hospital, Oncology Training and Research Hospital, Medical University of Graz - Graz, Áustria.

Financial Support: None.

Conflict of Interest: None. 
improving survival chances.

Dermoscopy is a noninvasive technique that helps distinguish melanomas from other pigmented and non-pigmented skin lesions. It provides information about skin structures in the epidermis, dermoepidermal junction and dermis via optical magnification and liquid immersion. ${ }^{6}$ Despite the advances in understanding of the dermoscopic features of extrafacial melanoma and their relationship to Breslow thickness, little is known about the dermoscopic features of HNM.

Stolz et al. and Pralong et al. have identified specific, dermoscopic criteria for facial lentigo maligna melanoma (LMM). ${ }^{7,8}$ Stolz et al. have described a progression model, consisting of four steps. As characterized by these researchers, the newly identified dermoscopic patterns include: hyperpigmented follicular openings, fine grey dots and globules, rhomboid structures, obliterated hair follicles, increased density of the vascular network, red rhomboidal structures.

The aim of this study was to provide a baseline definition of melanoma patterns in the head and neck region, and compare the frequencies of dermoscopic findings across the histological subtypes of HNM.

\section{METHODS}

This observational, descriptive study was conducted by three dermatologists and two ear, nose, and throat specialists. The dermoscopic photos of HNM seen at the four participating clinics (Department of Dermatology, Kars State Hospital, Kars; Department of Dermatology, Kutahya Tavsanli State Hospital, Kutahya; Department of Ear, Nose and Throat, Ankara Oncology Training and Research Hospital, Ankara; Department of Dermatology, Medical University of Graz) were included. All patients signed a consent form, allowing their photos to be used in the study. Inclusion criteria were: aged 18 or over, prior consultation in the department of dermatology office for suspicion of head and neck melanoma, and the presence of at least 1 head and neck melanoma diagnosed clinically, dermoscopically and histopathologically. Mucosal melanomas were excluded.

The samplefor this observational study consisted of 108 dermoscopic HNM images, captured with a Canon PowerShot A630 digital camera, equipped with a Dermlite Foto Dermascope. All dermoscopic images were evaluated by three observers. Clinical data, including sex, age, location of the lesion, skin type (ST) according to the Fitzpatrick scale, diameter and dermoscopic and histopathological findings were recorded for each participant.

In addition to classical dermoscopic features of extrafacial melanoma (asymmetry in 2 axes, atypical network, atypical dots, structureless areas, blotches, blue-white veil, radial streaming, pseudopods, pseudonetwork), we applied the classical Stolz criteria (asymmetric pigmented follicular openings, rhomboidal structures, annular-granular pattern, obliterated hair follicles) and new criteria defined by Pralong et al. (increased density of the vascular network, red rhomboidal structures). ${ }^{7,8}$ The 108 melanomas were then classified as lentigo maligna melanoma (LMM), superficial spreading melanoma (SSM), nodular melanoma, and desmoplastic melanoma on the basis of the dermatopathological diagnosis.

SPSS 15.0 (SPSS Inc., Chicago, IL, U.S.A.) was used in the statistical analysis with a statistical significance of $p<0.05$. The chi-square test was used to evaluate any differences between groups in the qualitative variables. For correlations between variables, Spearman correlation coefficients were estimated.

Global agreement on dermoscopic features was estimated by Cohen's kappa statistic. Kappa values of 0.4 and 1 indicate strong agreement, while values below 0.4 indicate poor agreement.

\section{RESULTS}

\section{Clinicopathologic Features}

A total of 108 head and neck melanomas in 108 patients were included (68 male and 40 female; the mean age, $64.56 \pm 15.01$ years). Out of these patients, $16(14.8 \%)$ had Fitzpatrick skin type I, $56(51.9 \%)$ had Fitzpatrick skin type II, 32 (29.6\%) had Fitzpatrick skin type III, and $4(3.7 \%)$ had Fitzpatrick skin type IV. The most frequent location for head and neck melanoma was the cheek (60 patients, $55.6 \%$ ), followed by the forehead (16 patients, $14.8 \%$ ), neck (12 patients, $11.1 \%$ ), nose (8 patients, $7.4 \%)$, scalp (8 patients, $7.4 \%$ ), and external ear (4 patients, 3.7\%). The median diameter was $10 \mathrm{~mm}$ (range 5-35).

The 108 HNMs consisted of 20 in situ lentigo maligna melanomas (18.5\%), 12 in situ superficial spreading melanomas (11.1\%), 40 lentigo maligna melanomas (37\%), 18 superficial spreading melanomas $(16.7 \%), 10$ nodular melanomas $(9.3 \%)$ and 8 desmoplastic melanomas (7.4\%), histopathologically. They included 32 cases of in situ melanoma (29.6\%), 48 cases of melanoma with Breslow thickness $<1 \mathrm{~mm}$ $(44.4 \%)$, and 28 cases of melanoma with Breslow thickness $>1 \mathrm{~mm}(26 \%)$.

\section{Dermoscopic Features}

The dermoscopic features observed in our series of 108 HNMs are shown in tables 1-2 and figures 1-3. In addition, the most frequent dermoscopic feature 
TABLE 1: Frequency of the analyzed criteria in head and neck melanomas

\begin{tabular}{lll}
\hline Dermoscopic Characteristic & $\mathbf{N}$ & $\%$ \\
\hline Asymmetry in two axes & $68(\kappa=1)$ & 63 \\
Atypical dots & $80(\kappa=1)$ & 74.1 \\
Radial streaming & $32(\kappa=0.68)$ & 29.6 \\
Pseudopods & $8(\kappa=0.92$ & 7.4 \\
Blue-white veil & $20(\kappa=1)$ & 18.5 \\
Mixed vascular pattern & $44(\kappa=0.92)$ & 40.7 \\
Scar-like depigmentation & $64(\kappa=0.87)$ & 59.3 \\
Rhomboidal Structures & $32(\kappa=0.85)$ & 29.6 \\
Atypical pigment network & $26(\kappa=0.92)$ & 24 \\
Pseudonetwork & $32(\kappa=0.84)$ & 29.6 \\
Asymmetric pigmented follicular openings & $56(\kappa=1)$ & 51.9 \\
Annular-granular pattern & $20(\kappa=0.72)$ & 18.5 \\
Colors (3 or more) & $40(\kappa=0.88)$ & 37 \\
Blotches & $8(\kappa=1)$ & 7.4 \\
Increased density of vascular network & $35(\kappa=0.95)$ & 32.4 \\
Red rhomboidal structures & $20(\kappa=0.60)$ & 18.5 \\
Abrupt demarcation & $11(\kappa=0.76)$ & 10 \\
\hline
\end{tabular}

The value $\kappa$ is used for kappa coefficient

TABLE 2: Dermoscopic features according to the histological subtypes of melanoma

\begin{tabular}{|c|c|c|c|c|c|c|c|}
\hline $\begin{array}{l}\text { Dermoscopic } \\
\text { Features }\end{array}$ & LMM & LMM-in situ & SSM & SSM-in situ & Desmoplastic & Nodular & $P$ value \\
\hline Asymmetry & $\begin{array}{l}24 \\
(60 \%) \\
(\kappa=1)\end{array}$ & $\begin{array}{l}12(60 \%) \\
(\kappa=0.65)\end{array}$ & $\begin{array}{l}17(94.4 \%) \\
(\kappa=0.85)\end{array}$ & $\begin{array}{l}7(58.3 \%) \\
(\kappa=0.83)\end{array}$ & $\begin{array}{l}4(50 \%) \\
(\kappa=0.72)\end{array}$ & - & $P=0.0001$ \\
\hline Pseudonetwork & $\begin{array}{l}24(60 \%) \\
(\kappa=0.80)\end{array}$ & $\begin{array}{l}8(40 \%) \\
(\kappa=0.70)\end{array}$ & $\begin{array}{l}5(27.7 \%) \\
(\kappa=0.95)\end{array}$ & $\begin{array}{l}1(8.3 \%) \\
(\kappa=1)\end{array}$ & - & - & $\mathrm{P}=0.0005$ \\
\hline Irregular dots & $\begin{array}{l}32(80 \%) \\
(\kappa=0.83)\end{array}$ & $\begin{array}{l}15(75 \%) \\
(\kappa=1)\end{array}$ & $\begin{array}{l}12(66.6 \%) \\
(\kappa=0.76)\end{array}$ & $\begin{array}{l}7(58.3 \%) \\
(\kappa=0.91)\end{array}$ & $\begin{array}{l}3(37.5 \%) \\
(\kappa=0.85)\end{array}$ & $\begin{array}{l}5(50 \%) \\
(\kappa=1)\end{array}$ & $\mathrm{P}=0.121$ \\
\hline $\begin{array}{l}\text { Scar-like } \\
\text { depigmentation }\end{array}$ & $\begin{array}{l}12(30 \%) \\
(\kappa=0.86)\end{array}$ & $\begin{array}{l}6(30 \%) \\
(\kappa=0.69)\end{array}$ & $\begin{array}{l}6(33.3 \%) \\
(\kappa=0.84)\end{array}$ & $\begin{array}{l}3(25 \%) \\
(\kappa=1)\end{array}$ & - & $\begin{array}{l}5(50 \%) \\
(\kappa=0.92)\end{array}$ & $\mathrm{P}=0.032$ \\
\hline $\begin{array}{l}2 \text { different } \\
\text { colors }\end{array}$ & $\begin{array}{l}32(80 \%) \\
(\kappa=1)\end{array}$ & $\begin{array}{l}15(75 \%) \\
(\kappa=0.83)\end{array}$ & $\begin{array}{l}9(50 \%) \\
(\kappa=0.83)\end{array}$ & $\begin{array}{l}11(91.6 \%) \\
(\kappa=1)\end{array}$ & - & $\begin{array}{l}1(10 \%) \\
(\kappa=1)\end{array}$ & $\mathrm{P}=0.005$ \\
\hline $\begin{array}{l}3 \text { different } \\
\text { colors }\end{array}$ & $\begin{array}{l}8(20 \%) \\
(\kappa=1)\end{array}$ & $\begin{array}{l}5(25 \%) \\
(\kappa=0.80)\end{array}$ & $\begin{array}{l}5(27.7 \%) \\
(\kappa=0.73)\end{array}$ & $\begin{array}{l}1(8.3 \%) \\
(\kappa=0.47)\end{array}$ & - & $\begin{array}{l}5(50 \%) \\
(\kappa=0.82)\end{array}$ & $\mathrm{P}=0.005$ \\
\hline $\begin{array}{l}4 \text { different } \\
\text { colors }\end{array}$ & - & - & $\begin{array}{l}4(22.2 \%) \\
(\kappa=0.74)\end{array}$ & - & - & $\begin{array}{l}4(40 \%) \\
(\kappa=0.92)\end{array}$ & $\mathrm{P}=0.0005$ \\
\hline
\end{tabular}

The value $\kappa$ is used for kappa coefficient

in 80 out of 108 (74.1\%) HNMs was irregular dots, followed by asymmetry (63\%).

Asymmetry was a statistically significant dermoscopic feature for SSM, compared with LMM, LMM-in situ, SSM-in situ, desmoplastic melanoma, and nodular melanoma $(p<0.001)$.

Comparing all the histological types of HNM, pseudonetwork was a more common feature in LMM than in other groups, and there was a statistically significant difference between all the groups $(p<0.001)$. Furthermore, there was no statistically significant difference between histological subtypes of HNM in terms of irregular dots $(p=0.121)$.

Regarding the frequency of scar-like depigmentation in all lesions, it was significantly higher in nodular melanoma than in the other HNM 


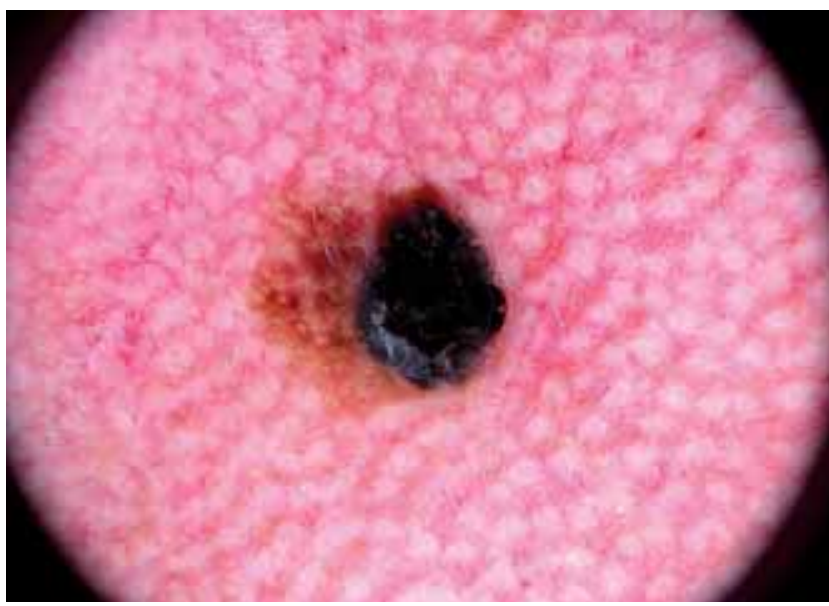

FiguRE 1: Superficial spreading melanoma with a Breslow index of $0.8 \mathrm{~mm}$ on the neck. Dermoscopy shows asymmetry in two axes, 3 colors, atypical pigment network, asymmetric pigment blotch, irregular streaks

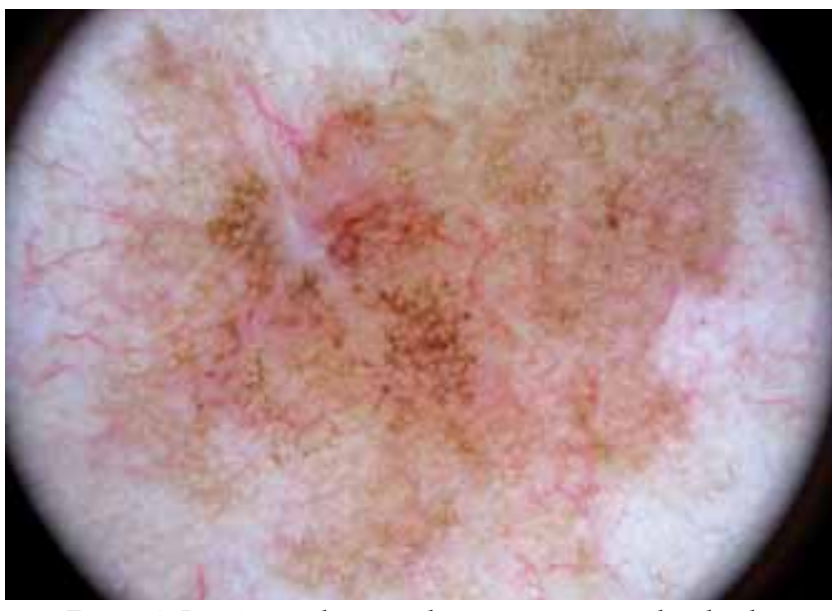

Figure 2: Lentigo maligna melanoma in situ on the cheek. Dermoscopy shows asymmetry in two axes, 3 colors, atypical dots, structureless area, scar-like depigmentation, atypical network

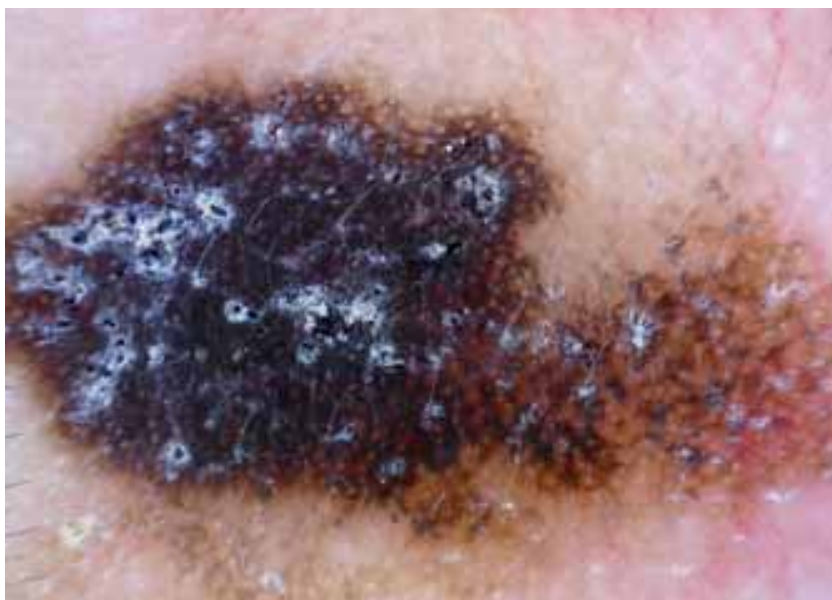

FigURE 3: Superficial spreading melanoma with a Breslow index of $1.3 \mathrm{~mm}$ on the cheek. Dermoscopy shows asymmetry in two axes, 4 colors, atypical network, streaks, atypical dots and globules, irregular vascularity. $(p=0.032)$.

Among the Stolz criteria for follicular invasion, asymmetric pigmented follicular openings were the most common feature, present in 56 cases $(51.9 \%)$, followed by rhomboidal structures in 32 cases $(29.6 \%)$, annular-granular pattern in 20 cases $(18.5 \%)$, and obliterated hair follicles in 9 cases $(8.3 \%)$.

Increased density of the vascular network was observed in 35 LMM and LMM-in situ cases (58.3\%). Red rhomboidal structures were found in $20 \mathrm{LMM}$ and LMM-in situ cases (33.3\%). Moreover, increased density of the vascular network and red rhomboidal structures were not noted in other types of melanoma.

The presence of 2 different colors was a more constant feature in LMM, LMM-in situ and SSM-in situ $(p=0.005)$ than in SSM and nodular melanoma $(p=0.001)(p=0.03)$. Regarding the presence of 3 different colors in all lesions, there was a statistically significant difference except when comparing nodular melanoma with other types of melanoma $(p=0.005)$. The presence of 4 different colors was a statistically significant feature in nodular melanomas, compared with other types of HNM ( $p<0.001)$.

Comparison between invasive and in situ HNM showed that the number of colors was mostly $\geq 3$ in invasive HNM (8 vs 32$)(p=0.042)$. Additionally, we found a positive correlation between the number of colors and Breslow thickness $(p<0.001, r s=+0.385)$. However, blue-white veil, radial streaming, irregular dots, annular granular structure, black blotches, asymmetrical pigmented follicular openings, scarlike depigmentation, mixed vascular patterns and the presence of asymmetry were not statistically significant for in situ or invasive HNM.

\section{DISAUSSION}

This study shows clinical results consistent with previous articles. In head and neck melanomas, the most affected site was the face, particularly the cheek. Extremity melanomas were observed in the younger age group, while head and neck melanomas were seen in the older age group. ${ }^{9,10}$

Cutaneous melanomas in the head and neck reportedly have a poorer prognosis than those occurring in the trunk and extremities. ${ }^{5}$ Ettl et al. demonstrated that melanomas in the face region have a better prognosis than melanomas arising in the scalp, ear and neck, within the head and neck area. ${ }^{10}$ They suggested that the reason for better prognosis may be a predominance of lentigo maligna melanoma in the face region as well as the predominance of nodular melanoma in the scalp, ear and neck. A further reason they advanced was early detection of facial melanoma. This study is the first to include the Stolz and Pralong criteria to investigate dermoscopic characteristics, 
drawing on a large sample size. Our data substantiate the premise that the general dermoscopic features of trunk or extremity melanoma cannot be applied to facial localizations and suggest that familiarity with face-specific, dermoscopic hallmarks is needed to detect head and neck melanomas at early stages.

Stratum corneum of facial skin is thinner than that of the trunk and limbs, while pilosebaceous units and sweat glands are densely present in the facial skin. The thin epidermis allows blood vessels or melanophages to be observed more easily on facial skin than elsewhere on the extrafacial skin. In addition, the pseudonetwork and sun-damaged elastosis are often present. Facial nevi are usually characterized by unpigmented or poorly pigmented, elevated lesions, which correspond histopathologically to the dermal nevi. For similar reasons, specific dermoscopic features have been described for melanomas on the face.

Stolz et al. have identified a "progression model for lentigo maligna", comprising four steps of lentigo maligna melanoma invasion of the hair follicles, by dermoscopic examination. ${ }^{7}$

The first change is regular/irregular, hyperpigmented follicular openings corresponding to the earliest invasion of the hair shaft histopathologically. Subsequently, the signet-ring-shaped structures (thickening of the hyperpigmented follicular openings) and annular-granular pattern (multiple, grey dots and globules around the pilo-sebaceous units) appear with further invasion. Furthermore, pigmented, rhomboidal structures (lozenge-shaped) are created in the perifollicular area while progressing. Finally, the follicular lumen is completely covered with uniformly pigmented areas.

In our study, the criteria of Stolz et al. were observed in LMM-in situ and LMM, of which asymmetric pigmented follicular openings was the most common (present in about 8 in $10 \mathrm{LMM}$ or LMM-in situ cases). Hence, the presence of this feature identified by Stolz et al. should be investigated for early diagnosis of lentigo maligna melanoma, as recommended in the literature. ${ }^{11}$

Braun et al. demonstrated the importance of irregular dots when analyzing the dermoscopic granularity of extrafacial melanoma. ${ }^{12}$ According to their results, granularity was observed in $26.5 \%$ of benign lesions and $93.5 \%$ of melanomas, in extrafacial melanomas. Granularity located at the edge of lesions was linked to the diagnosis of melanoma on extrafacial skin. Foci of multiple blue or gray dots, often described as "pepper-like" granules, can appear as fine granules or as coarse granules. These dots were observed in $74.1 \%$ of cases, with no statistically significant difference between groups in our study. In accordance with the results, it appears that the frequencies of irregular dots in facial melanomas are lower than in extrafacial melanomas.

The number of colors present can be used to predict melanoma thickness. ${ }^{13}$ In our study, in situ LMMs, in situ SSM, and LMMs were more often bicolored and always presented with $<4$ standardized, dermoscopic colors. In contrast, in our study, SSM nodular melanomas presented with 3 or more standardized, dermoscopic colors. According to the results obtained by Pralong et al., in situ LMMs were more often associated with 1 or 2 colors, while invasive LMM tends to have 5 or more colors. ${ }^{8}$ Our results support the findings of Pralong et al. It was demonstrated that all malignant melanomas show 3 or more colors in $85 \%$ of cases. ${ }^{14}$ The results lead us to suggest that facial melanomas more frequently entail 2 colors or less, compared to extrafacial melanoma.

Since the dermo-epidermal junction is flat on the face, a typical pigment network (honeycomb-like pattern) can barely be found. The pseudonetwork is formed by the numerous, round, pigment-free network holes corresponding to the openings of hair follicular ostia and adnexal structures. A pseudonetwork can be found in seborrhoeic keratosis, pigmented actinic keratosis, lichen planus-like keratosis, in addition to lentigo maligna. In our study, a pseudonetwork was observed in 30\% of LMM cases, $40 \%$ of in situ LMM cases, and $61.1 \%$ of SSM cases. Results obtained by Goncharova et al. demonstrated that a pseudonetwork was present in $87.5 \%$ of lentigo maligna cases, $52.94 \%$ of actinic keratosis (AK) cases, and $40 \%$ of solar lentigo cases. ${ }^{15}$ Meanwhile, Akay et al. found pseudonetworks in $36 \%$ of facially pigmented AK. ${ }^{16}$ Although melanophages accumulate in pigmented AK and present as gray dust, similarly to lentigo maligna, in LMM, the structure of the pseudonetwork is characterized by an accumulation of neoplastic melanocytic cells in hair follicles. ${ }^{17}$

Pralong et al. described two new, vascularly linked dermoscopic features in LMM. An increased vascular network corresponds to in situ stages of LMM during a histopathologic analysis. Moreover, red rhomboidal structures, lozenge-shaped vascular structures in the perifollicular area, were correlated to tumor invasion in LMM. They found an increased density of the vascular network in $58 \%$ of LMM cases and red rhomboidal structures in $40 \%$ of LMM cases. ${ }^{8}$ In our case series, an increased density of the vascular network was present in 58.3\% of LMM patients, while red rhomboidal structures were present in 33.3\% of LMM patients. These data are similar to those reported by Pralong et al.. The detection of vascular characteristics may be helpful, particularly the general, pigmented, dermoscopic features are not present. Further studies on larger populations are needed to confirm these diagnostic, vascular, dermoscopic 
criteria.

Among regression-associated dermoscopic features, scar-like depigmentation was observed in $30 \%$ of LMM and in situ LMM cases, as well as in $50 \%$ of nodular melanoma patients. In terms of scarlike depigmentation, there is no difference between facial and extrafacial melanomas, and it is much more common in invasive melanomas.

Furthermore, classical extrafacial melanoma, vertical growth phase-associated, dermoscopic features were barely found: abrupt demarcation in $10 \%$ of cases, atypical pigment network in $24 \%$ of cases, blue-white veil in $18.5 \%$ of cases, radial streaming in $29.6 \%$ of cases, pseudopods in $7.4 \%$ of cases, and irregular blotches in only $7.4 \%$ of cases. These characteristics are associated with poor prognoses in patients with invasive melanomas.

\section{CONQLUSION}

HNM, particularly LMM, has specific dermoscopic features, and classical, extrafacial, dermoscopic rules are less useful for diagnosizing facial melanoma. The criteria applied by Stolz et al. and
Pralong et al. are supported by this study. Our results lead us to suggest that low frequencies of irregular dots, 2 or fewer colours in lesions, increased density of the vascular network, red rhomboidal structures, the presence of pseudonetworks, asymmetric pigmented follicular openings, signet-ring-shaped structures, and the annular-granular pattern are head- and neckspecific, dermoscopic features. Moreover, classic, invasive, melanoma-associated, dermoscopic features such as blue-white veils, irregular blotches, scar-like depigmentation, radial streaming and pseudopods are less common in facial melanomas.

Although there were no statistically significant differences as regards blue-white veils, radial streaming, irregular dots, annular granular structures, black blotches, asymmetrical, pigmented follicular openings, scar-like depigmentation, mixed vascular patterns and the presence of asymmetry between in situ and invasive melanomas, there was an association between the number of colors and Breslow thickness. Further prospective studies of a larger number of patients and of head and neck melanomas will provide more definite results.

\section{REFERENCES}

1. Alex JC. The diagnosis and management of cutaneous melanoma of the head and neck, Presentation and Handout, Annual Meeting. American Academy of Otolaryngology Head and Neck Surgery: New Orleans; 1999.

2. Bodenham DC. Malignant melanoma of the head and neck. Excerpta Medica. 1975:85-91.

3. Barnes L. Surgical Pathology of the Head and Neck. 2nd ed. Vol. 3, Skin lesions of the head and neck. New York: Marcel Dekker; 2001. p. 1819-24.

4. Franklin JD, Reynolds VH, Bowers DG Jr, Lynch JB. Cutaneous melanoma of the head and neck. Clin Plast Surg. 1976;3:413-27.

5. Fadaki N, Li R, Parrett B, Sanders G, Thummala S, Martineau L, et al. Is head and neck melanoma different from trunk and extremity melanomas with respect to sentinel lymph node status and clinical outcome? Ann Surg Oncol. 2013;20:3089-97.

6. Braun PR, Robinovitz SH, Oliviero M, Kopf WA, Saurat H-J, Thomas L. Dermoscopic Examination. In: Soyer HP, Argenziano G, Hofmann-Wellenhof R, Johr R, editors. Color Atlas of Melanocytic Lesions of the Skin. Berlin: SpringerVerlag; 2007. p. 7-22.

7. Stolz W, Schiffner R, Burgdorf WH. Dermatoscopy for facial pigmented skin Iesions. Clin Dermatol. 2002;20:276-8

8. Pralong P, Bathelier E, Dalle S, Poulalhon N, Debarbieux S, Thomas L. Dermoscopy of lentigo malignant melanoma: report of 125 cases. Br J Dermatol. 2012;167:280-7.

9. Egger ME, Tabler BL, Dunki-Jacobs EM, Callender GG, Scoggins CR, Martin RC 2nd, et al. Clinicopathologic and survival differences between upper and lower extremity melanomas. Am Surg. 2012;78:779-87.

10. Ettl T, Irga S, Müller S, Rohrmeier C, Reichert TE, Schreml S, et al. Value of anatomic site, histology and clinicopathological parameters for prediction of lymph node metastasis and overall survival in head and neck melanomas. J Craniomaxillofac Surg. 2014;42:e252-8.

11. Stante M, Giorgi V, Stanganelli I, Alfaioli B, Carli P. Dermoscopy for early detection of facial lentigo maligna. Br J Dermatol. 2005;152:361-4.
12. Braun RP, Gaide 0, Oliviero M, Kopf AW, French LE, Saurat JH, et al. The significance of multiple blue-grey dots (granularity) for the dermoscopic diagnosis of melanoma. Br J Dermatol. 2007:157:907-13.

13. Stante M, De Giorgi V, Cappugi P, Giannotti B, Carli P. Non-invasive analysis of melanoma thickness by means of dermoscopy: a retrospective study. Melanoma Res. 2001;11:147-52.

14. Stolz W, Braun-Falco O, Bilek P, Landthaler M, Burgdorf W, Cognetta A. Atlas Colorido de Dermatoscopia. 2 ed. Rio de Janeiro: DiLivros; 2002.

15. Goncharova Y, Attia EA, Souid K, Vasilenko IV. Dermoscopic features of facial pigmented skin lesions. ISRN Dermatol. 2013;2013:546813.

16. Akay BN, Kocyigit P, Heper AO, Erdem C. Dermatoscopy of flat pigmented facial lesions: diagnostic challenge between pigmented actinic keratosis and lentigo maligna. Br J Dermatol. 2010;163:1212-7.

17. Schiffner R, Schiffner-Rohe J, Vogt T, Landthaler M, Wlotzke U, Cognetta AB, et al. Improvement of early recognition of lentigo maligna using dermatoscopy. J Am Acad Dermatol. 2000;42:25-32.
M AILING ADDRESS:
Fatma Pelin Cengiz
Bezmialem $V$ akif U niversity, Faculty of M edicine
$D$ epartment of D ermatology
34093 Fatih
Istanbul, Turkey.
E-mail: fpelinozgen@hotmail.com

How to cite this article: Cengiz FP, Cengiz AB, Emiroglu N, Comert E, Hofmann-Wellenhof R. Dermoscopic and clinical features of head and neck melanoma. An Bras Dermatol. 2015;90(4):488-93. 